\title{
Introduction to the 2016 Peter J. Buckley and Mark Casson AIB Dissertation Award
}

\author{
Anupama Phene, George Washington University, USA
}

THIS YEAR'S SUBMISSIONS to the Peter J. Buckley and Mark Casson AIB Dissertation Award offered an exciting look at the thoughtful, innovative, and interesting manner in which doctoral students are exploring and extending the international business literature. Submissions were invited from doctoral students who successfully defended their dissertations during 2015 on a topic that contributes to international business. As the award has evolved, it has recognized the seminal contributions made by its eponymous scholars. Previously known as the Richard N. Farmer Dissertation Award, it emphasizes past AIB President Richard Farmer's holistic approach to understanding the environment of international business. In 2013, with new sponsorship, the award recognizes Peter J. Buckley and Mark Casson for their pioneering work on internalization theory and the existence of the multinational enterprise. Consequently, the call for submissions highlights the joint importance of the environment of international business and the nature of the multinational enterprise. All of the finalist dissertations incorporate both of these aspects.

\section{Selection Process}

A total of 29 submissions were received and diligently evaluated by a knowledgeable committee including Sumit Kundu, Shige Makino, Rebecca Piekkari, and chaired by myself. The submissions encompassed a broad spectrum in terms of theoretical approaches, empirical contexts, and international business questions and phenomena explored. In a fitting representation of the field of international business, the geographic dispersion of the candidates' affiliations represented a wide variety of locations across the globe, including Australia, Bangladesh, Belgium, Chile, China (and Hong Kong), Denmark, Finland, France, India, Italy, Norway, Sweden, UAE, UK, and the US.

The quality of submissions received made the selection process challenging. The committee used the following criteria to determine the finalists as well as the eventual award winner: relevance and contribution to the field of international business, quality of the theoretical framing and the methodology, and quality of the empirical work (where relevant). Each committee member used her/his judgement to assess the initial submissions on these criteria and selected a ranked set of the top ten submissions. We then compiled the rankings on the basis of both the total score received as well as the average score received by candidates ranked by three or more committee members. Both of these methods resulted in the identification of three submissions with the highest scores. For the fourth position, the methods resulted in the identification of three more submissions with scores that were similar to each other. Each committee member then re-assessed these three submissions and ranked them to identify a fourth finalist. These finalists were as follows (listed in alphabetical order, with the award winning dissertation mentioned first):

Hetereogenous implementation of CSR in an MNE: The role of subsidiaries' institutional contexts and behaviors by Anne Servantie Jacqueminet, Bocconi University (Ph.D. awarded by Ecole des Hautes Etudes Commercials de Paris)

Influence of institutional and geographical factors on the openness and dispersion of knowledge- sourcing practices by Marcelo Fabian CanoKollman, Ohio University (Ph.D. awarded by Temple University)

Influences on transfer effectiveness: An exploratory study of headquarters transfer of capabilities to subunits in the multinational corporation by Olof Lindahl, Uppsala University (Ph.D. awarded by Uppsala University

Language strategies in multinational corporations: A cross-sector study of financial service companies and manufacturing companies by Guro R. Sanden, Copenhagen Business School (Ph.D. awarded by Copenhagen Business School)

The dissertations continue to reflect the broadening of the scope of international business, encompassing topics related to corporate social responsibility, language strategy, connectivity of peripheral economies, and exploration of the role of headquarters in the multinational firm, supporting the trends observed by Rugman (2013) and Buckley (2014). These finalists were invited to submit complete dissertations that were then read and ranked by the entire committee using the criteria indicated earlier. The winner, Anne Jacqueminet, was selected based on the compiled rankings. The committee's work was admirably supported by the AIB administration, notably Tunga Kiyak's assistance was much appreciated.

\section{Takeaways for Future Aspirants}

After participating in evaluating dissertation submissions over the last few years, these are some of my thoughts on what makes a good disser- 
tation. For new students embarking on their doctoral programs, I hope they are helpful in creating a foundation for a strong research portfolio.

\section{Ask interesting and innovative questions that extend our under-} standing of international business

Each of this year's finalists demonstrates this trait in their work, exploring distinctive phenomena by asking innovative questions. Jacqueminet explores what makes MNE subsidiaries implement CSR practices that are consistent with headquarters $(\mathrm{HQ})$ policy, while Lindahl examines how HQ can facilitate the transfer of a common innovation capability across the firm's R\&D subsidiaries. Cano-Kollman considers how national policies and support influence firm innovation, and Sanden evaluates how language management tools are implemented in multinational firms across industry sectors. Posing the right questions allows students to conduct pioneering work in new creative fields (Buckley, 2014) and engage in novel research that will support long term ambition and a long lived career (Casson, 2015).

\section{Root these questions in strong theory}

The finalists posed innovative questions and they also explored these questions while anchoring them in robust theoretical foundations. Without theoretical support, a dissertation can become phenomenon-driven and pose greater challenges to publish, in the long term. Anchoring phenomena in theory requires a good understanding of existing theories since it also involves questioning them or integrating different theories. Identifying exceptions to theoretical predictions by considering boundary conditions or reconciling the differing implications of theories are good ways to think about how international business theory can be extended. Use the doctoral years to hone understanding of the different theories so that it is possible to contribute to extending them.

\section{Use unique data or use archival data creatively in conjunction with sophisticated empirical analysis}

Several of the finalists demonstrated efforts to collect unique data through interviews and case studies. Others combined archival data in creative ways to explore their questions. All finalists utilized cutting edge empirical techniques to test their hypotheses. This is in keeping with the trend in international business research, with more rigorous methods becoming the norm. As a doctoral student, invest the time in data collection efforts and learning new methodologies.

\section{Conclusion}

As evidenced by the excellent quality of the finalist dissertations, it is clear that the bar to become a finalist for the Buckley and Casson award is extremely high. Congratulations to the winner and all the finalists! On behalf of the committee, I wish you the very best in your research endeavors. We hope to read more of your impactful work as you expand the horizons of international business.

\section{References}

Buckley, P. 2014. Introduction to the Special Issue on the 2014 Peter J. Buckley and Mark Casson AIB Dissertation Award. AlB Insights, 14(3): 4.

Casson, M. 2015. The Buckley and Casson Prize: Some reflections. AlB Insights, 15(3): 3-5.

Rugman, A. 2013. From the Richard N. Farmer to the Peter J. Buckley ad Mark Casson AIB Dissertation Award. AlB Insights, 13(3): 4-5.

Anupama (Anu) Phene (anuphene@gwu.edu) is a Professor of International Business and Phillip Grub Distinguished Scholar at the George Washington University School of Business. Her research focuses on multinational firm and subsidiary innovation, knowledge transfer within and across firms, mechanisms to transfer knowledge and the geographic boundaries of knowledge. She has published articles in Administrative Science Quarterly, Strategic Management Journal, Organization Science, Journal of International Business Studies, Management International Review, and the Journal of Management. 